\title{
BACKGROUND-LIMITED PERFORMANCE IN THE SUBMILLIMETER
}

\author{
J. A. DAVIDSON AND T. L. ROELLIG \\ NASA/Ames Research Center, MS 245-6 \\ Moffett Field, CA 94035-1000, USA \\ R. J. PERNIC \\ Yerkes Observatory \\ Williams Bay, WI 53191, USA
}

\section{Introduction}

We have been awarded NASA funds to construct a Submillimeter (SMM) Array Photometer for use at the Caltech SMM Observatory (CSO) on Mauna Kea. The array will consist of $5 \times 5$ composite bolometers illuminated through light cones. On the CSO, each cone will cover 25 arcsecs on the sky, implying a total field-of-view for the array of just over $2 \times 2$ arcmins. Our aim is to achieve background-limited performance in the 730 and $870 \mu \mathrm{m}$ atmospheric windows, thus obtaining a Noise Equivalent Flux Density (NEFD) of approximately $0.1 \mathrm{Jy} / \mathrm{JHz}$ at these wavelengths. This sensitivity level will enable 5-sigma measurements of $10 \mathrm{mJy}$ sources (such as radioquiet quasars) in about one hour. We also will have the capability to operate at $1.1 \mathrm{~mm}$, but the photometer will not be optimized at this wavelength. This system is expected to be operational on the CSO in January 1994.

\section{Present Sensitivity Performances}

At present we have achieved an NEFD of $2 \mathrm{Jy} / \mathrm{JHz}$ at the CSO using a 7-bolometer, $\mathrm{He}^{3}$-cooled photometer operating with a filter which covers both the 730 and $870 \mu \mathrm{m}$ atmospheric windows. Clearly this system is not background-limited and is probably detector-limited. Currently, only the CSO and the James Clerk Maxwell Telescope (JCMT) have facility operated submillimeter photometers on Mauna Kea, and both of these are single bolometer systems. Each of the seven channels in our system has a sensitivity comparable to these photometers. At $800 \mu \mathrm{m}$ the CSO and JCMT photometers achieve $1-5$ and $0.5-1 \mathrm{Jy} / \mathrm{JHz}$, respectively. The range of sensitivities for a given telescope is due to the atmosphere. Much of the difference between the sensitivities of the CSO and JCMT photometers can be explained by the difference in telescope diameters; the JCMT is a 15 meter telescope and the CSO telescope is 10 meters in diameter. Our NEFD goal, as stated above, is based on the assumption that with our new system we will be backgroundlimited, not detector limited. This sensitivity goal was calculated using the telescope parameters for the CSO, and the sky and telescope background Noise Equivalent Powers (NEP's) for an atmospheric water vapor value of $1.0 \mathrm{~mm}$. An NEFD of $0.1 \mathrm{Jy} / \mathrm{JHz}$ will require an improvement of a factor of 20 over that achieved using our 7-bolometer system. Such an improvement is possible if the optical efficiency of the 7-bolometer system were increased by a factor of three and the detector NEP were reduced by a factor of seven. The first requirement will be relatively straightforward to achieve with the replacement of a few of our present filters which are particularly inefficient. 
The second requirement could be met by cooling our proposed array of 25 bolometers to a temperature of $0.05 \mathrm{~K}$, one seventh the operating temperature of our present system, because the NEP of a bolometer is proportional to its operating temperature.

One source of noise not taken into account in the above background NEP discussion is that noise generated by the changing emissivity of the atmosphere, called sky-noise. This varies from night to night and can sometimes be very severe, increasing the effective NEFD of a system by many factors. Based on our observations with the 7-bolometer photometer, sky-noise is usually correlated across the array. Therefore, by using an array, one hopes to be able to reduce the effects of sky-noise in the final reduced data. We are currently taking sky-noise data so that we can characterize it in order to (1) improve data acquisition methods in the presence of such noise, and (2) model sky noise for subtraction purposes.

\section{The Photometer}

In order to achieve the low temperatures required $(0.05 \mathrm{~K})$, we are constructing an Adiabatic Demagnetization Refrigerator (ADR) to cool the 25bolometer array. The whole photometer will be contained in a cylindrical dewar, 26 inches in length and 14 inches in diameter. The bulk of this volume will be employed for housing the liquid nitrogen and helium reservoirs, which will maintain the photometer's cold work surface at $4 \mathrm{~K}$ for well over 24 hours and provide thermal shielding. The heart of the ADR photometer is a paramagnetic salt-pill which lies in the core of a superconducting magnet. The superconducting magnet is attached to the $4 \mathrm{~K}$ work surface of the photometer. Our design suspends the salt-pill via a $\mathrm{He}^{3}$-cooled support structure which operates at a temperature of $0.3 \mathrm{~K}$. This design allows operation of the photometer on the telescope without pumping on the $\mathrm{He}^{4}$ reservoir (Ruhl and Dragovan, 1991). The salt-pill (which has been constructed) is made from Ferric Ammonium Sulfate crystals grown around gold wires which will lead to the bolometer array. Such a salt-pill, after complete demagnetization, can maintain a temperature of $0.05 \mathrm{~K}$ for 60 hours with a heat load of $90 \mathrm{nW}$. This load is about half that expected for the 25bolometer array.

The optics of the photometer will be very simple, since the photometer will mount to the CSO telescope via a mounting box, containing relay optics which reimage the F12.36 Cassegrain focus to one of two F4.4 foci, by means of a flat steering mirror. The elliptical mirror in the relay optics forms an image of the primary as well as an image of the Cassegrain focus. These images are exploited in our design for the photometer where we have placed our cold pupil stop and filter wheel at the image of the primary (inside the dewar) and the entrance of our cones in the bolometer array at the F4.4 focus. The cones in the array will be tilted inwards to illuminate the pupil stop only. Calculations show that for secondary mirror chop throws up to \pm 3 arcmins and individual beamsizes of 25 arcsec, the image quality will be good (Strehl ratio $>0.9$ ) over the whole array.

\section{REFERENCES}

Ruhl, J. and Dragovan, M., 1991. Low Temperature Detectors for Neutrinos and Dark Matter, ed. Booth, N.E., and Salmon, G.L., Conf. Proc. 4, 461. 